\title{
Development of Monochromatic Analytical Electron Microscope Equipped with Higher-Order Aberration Corrector
}

\author{
Masaki Mukai, Eiji Okunishi, Akiho Nakamura, Takaki Ishikawa and Hidetaka Sawada \\ JEOL Ltd., 3-1-2 Musashino, Akishima, Tokyo 196-8558, Japan \\ * Corresponding author: mmukai@jeol.co.jp
}

Monochromatic and aberration corrected electron microscope was developed in order to study an electronic structure of various materials, combining with a scanning transmission electron microscope (STEM) and an electron energy-loss spectroscopy (EELS) at atomic scale. [1] This microscope is equipped with a double Wien-filter monochromator, and obtained energy resolutions are selectable from $0.25 \mathrm{eV}$ to $0.02 \mathrm{eV}$ keeping the atom-sized electron probe on the specimen plane by the change of the width of the slit located between two filters. Since this microscope is equipped with the conventional thirdorder aberration corrector, the residual dominant geometric aberration was six-fold astigmatism. On the other hand, the higher-order aberration corrector was developed, which enable us to correct fifth-order geometric aberrations including six-fold astigmatism and to expand a uniform phase area of a Ronchigram in STEM to about $60 \mathrm{mrad}$ in half angle even at lower accelerating voltages. [2-3]

We have developed a new monochromated analytical electron microscope equipped with the higher-order aberration corrector. Figures 1(a) and (b) show comparison of the Ronchigrams at $200 \mathrm{kV}$, when we used the higher-order and conventional third-order aberration correctors. Due to the correction of six-fold astigmatism by the higher-order corrector, the uniform phase area in the Ronchigram was extended to be $60 \mathrm{mrad}$ as shown in Fig. 1(a). Figures 2(a), (b) and (c) show the raw high-angle annular dark-field (HAADF)-STEM images and their power spectra for sample of Si «110〉, obtained with monochromatic electron probes. The convergent semi-angles of the probe were ranged from $19.8 \mathrm{mrad}$ to $47.3 \mathrm{mrad}$, and energy spread of that was $0.25 \mathrm{eV}$. The $\{440\}$ reflections, which implies lattice spacing of $96 \mathrm{pm}^{-1}$, are present in the all power spectra of the recorded images, owing to correction of six-fold astigmatism by the higher-order aberration corrector and reduction of chromatic aberration by the monochromator. Realization of the atomic sized electron probe with a large convergence angle by the monochromator and the higher-order aberration corrector suggests the possibility of the optical sectioning at sub-nanometer depth resolution in STEM. [4]

Figures 3(c)-(f) show the simultaneously acquired elemental maps of $\mathrm{SrTiO}_{3}\langle 100$ 〉 by EELS and energy dispersive X-ray spectroscopy (EDS) at $200 \mathrm{kV}$, where the probe current and the dwell time per a pixel were $120 \mathrm{pA}$ and 0.01 seconds. The obtained elemental maps of EELS and EDS exhibit atomic resolutions. Total solid angle for the EDS maps using two $100 \mathrm{~mm}^{2}$ sized silicon drift detectors (SDD) was $1.75 \mathrm{sr}$. and their take-off angles of the SDD detectors were $21.5^{\circ}$ and $18.3^{\circ}$. These results show high capability of the atomic resolution elemental analyses by EELS and/or EDS with the monochromatic electron microscope equipped with higher-order aberration corrector.

\section{References}

[1] M. Mukai, et al.: Microscopy, 64[3], (2015), 151-158.

[2] H. Mueller, et al.: Microsc. Microanal., 12 (2006), 442-455.

[3] T. Ishikawa, et al.: Microsc. Microanal., 13 (supple.3), (2015) 1599-1600

[4] S. Pennycook : Ultramicroscopy, 180 (2017), 22-33 

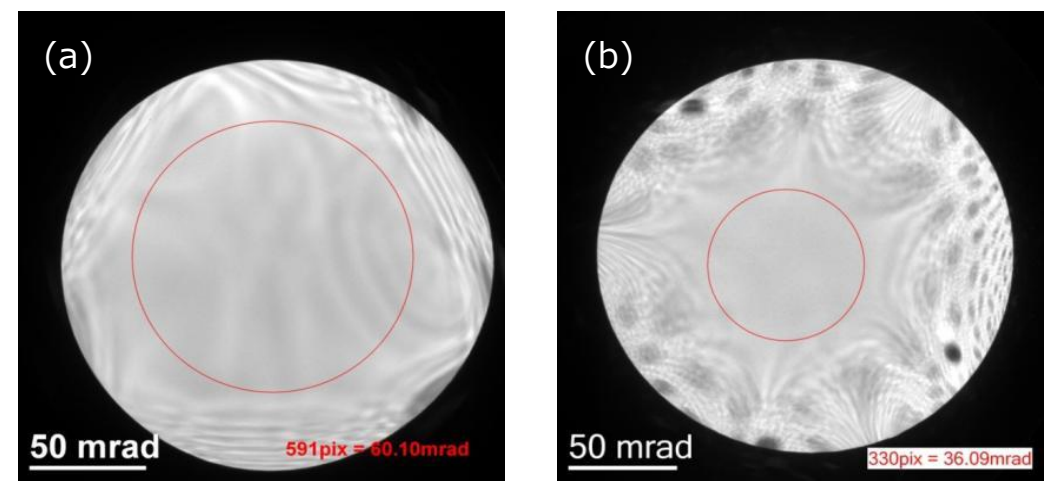

Figure 1. Comparison of the Ronchigrams at $200 \mathrm{kV}$, when we used (a) higher-order and (b) conventional third-order aberration correctors. The uniform phase areas in the Ronchigrams were (a) $60 \mathrm{mrad}$ and 36 mrad in semi angle. The coefficients of six-fold astigmatism were (a) $122 \mu \mathrm{m}$ and (b) $1500 \mu \mathrm{m}$.
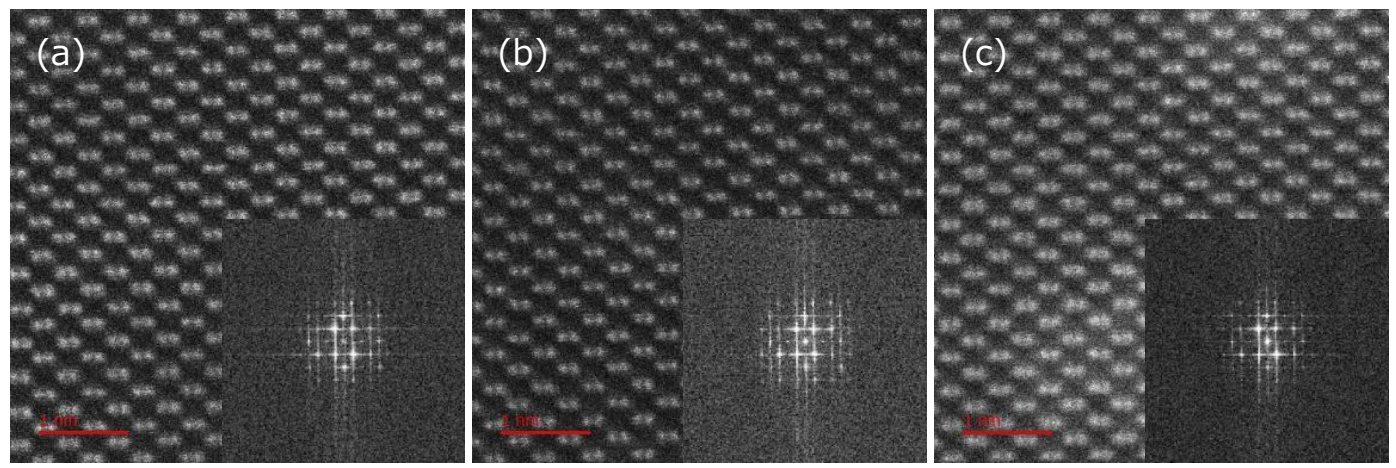

Figure 2. Comparison of raw HAADF-STEM images and their power spectra of Si $<110$ at $200 \mathrm{kV}$ on the convergent semi-angles of (a) $19.8 \mathrm{mrad}$, (b) $33.6 \mathrm{mrad}$ and (c) $47.3 \mathrm{mrad}$. The energy spread of the monochromatic probe was $0.25 \mathrm{eV}$, and the probe currents were (a) $8 \mathrm{pA}$, (b) $21 \mathrm{pA}$ and (c) $41 \mathrm{pA}$.
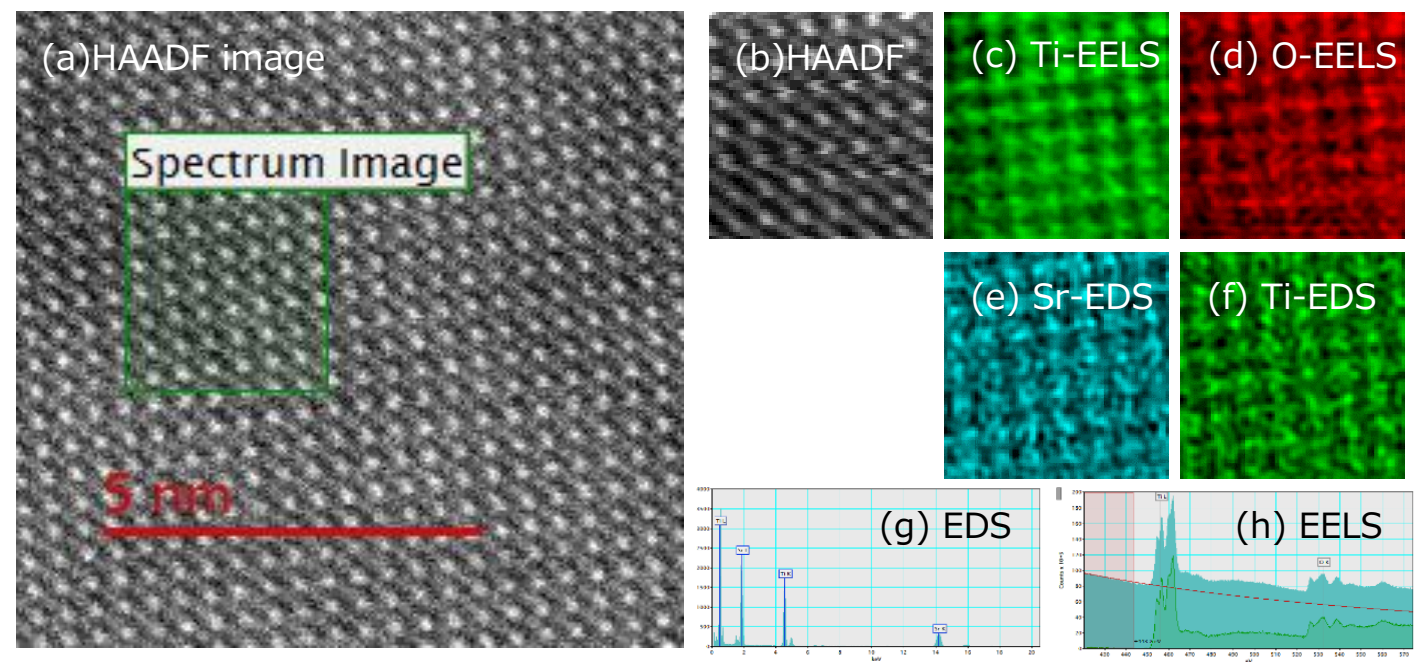

Figure3. Results of the simultaneous acquisition of atomic resolution HAADF images and elemental maps by EELS and EDS of $\mathrm{SrTiO}_{3}\langle 100$ 〉 at $200 \mathrm{kV}$, obtained with an electron probe of 120 pA probe current. (a) Raw HAADF-STEM image, (b) Raw HAADF-STEM image of the mapping area, the elemental maps of (c) Ti and (d) O obtained by EELS, (e) Sr and (f) Ti obtained by EDS and the spectra of (g) EDS and (h) EELS with an acquisition time of 0.01 seconds 